\title{
Visually-evoked cortical responses and pattern vision in the infant: A longitudinal study ${ }^{1}$
}

\author{
M. RUSSELL HARTER and CONSTANCE \\ D. SUITT, University of North Carolina, \\ Greensboro, N.C. 27412
}

Visually evoked cortical potentials were obtained from a human infant (from 21 to 155 days of age) in response to checkerboard-patterned light flashes (individual checks subtending from 20 to $133 \mathrm{~min}$ of arc). The largest amplitude cortical responses were evoked by relatively large checks during the first month of life and progressively smaller checks as the infant matured. The cortical response to the various check sizes was interpreted as reflecting the development of the visual system and a general improvement in visual actuity with age.

Data have been presented indicating that visually evoked cortical responses (VERs) to patterned light flashes may be used to study pattern vision in adult humans (Harter \& White, 1968, 1969; Rietveld, Tordoir, Hagenouw, Lubbers, \& Spoor, 1967; Spehlmann, 1965). This technique would be particularly useful in studying pattern vision in infants since motor responses are not required. Although VERs in infants have been investigated extensively using blank or diffuse light flashes (Ellingson, 1960, 1964; Engel, 1967; Engel \& Benson, 1968; Ferriss, Davis, Dorsen, \& Hackett, 1967: Hrbck, Vitova, \& Mares, 1966), they have not been investigated using patterned light flashes. The purpose of the present study is to investigate the effects of checkerboard-patterned light flashes on VERs in an infant.

\section{METHOD}

An intensive longitudinal investigation was performed on one full-term $(2,922 \mathrm{~g})$, normal male infant. He was born January 23,1969 , and, due to technical problems, was 21 days old before we obtained usable VER data from him. This report contains data collected up to the 156th day of life.

Visually evoked cortical responses were recorded monopolarly by means of a single gold-cup scalp electrode taped $1 \mathrm{~cm}$ above the inion on the midline. The roference electrode was attached to the right earlobe. Cortical activity was amplified by a Grass Model 7WC polygraph with high and low one-half amplitude frequency filters set at 35 and $3 \mathrm{~Hz}$, respectively. Activity occurring during the $512 \mathrm{msec}$ following each stimulus presentation was recorded. Both the signal-averaging and superimposition techniques were used to obtain VERs. Cortical responses were averaged by a Fabri-tek Model 1062 Instrument Computer and were recorded on graph paper with a Hewlett-Packard Model 7035B X-Y recorder. Superimposed tracings of individual VERs were recorded and photographed with a Hewlett-Packard Model 141A oscilloscope and a 196B oscilloscope camera. All data were recorded on an Ampex Model SP-300 seven-channel FM tape recorder. Electroencephalograms (EEGs) were monitored for movement and other artifacts by visual inspection of the polygraph chart record.

The stimuli were transparencies consisting of four checkerboard patterns (individual checks subtending visual angles of $20,43,86$, and $133 \mathrm{~min}$ of arc) and a "blank" pattern (B). These patterns were located $63 \mathrm{~cm}$ from the S's eyes, and their boundaries subtended a visual angle of $22 \times 22 \mathrm{deg}$. The patterns were placed over a translucent screen that covered a window at one end of a large box. A Grass Model PS-2 strobe lamp was mounted on the opposite end of the box, $100 \mathrm{~cm}$ distant from the rear of the window. A 10 microsec light flash was presented once every $1.1 \mathrm{sec}$ that evenly illuminated the rear of the translucent screen and thus back-illuminated the stimulus pattern. The photo-stimulator intensity setting was placed on " 4 " and " 2 " for the checkerboard- and blank-pattern conditions, respectively. This held luminous transmittance constant for the checkerboard and blank patterns.

The experiment was conducted in an electrically shielded, partially soundproof room. The $S$ was placed in a supine position, with his head turned sideways and oriented toward the stimulus. The room was darkened except for a dim light, placed above and behind the $S$, which enabled the $\mathrm{E}$ to monitor the orientation and movement of the S's head. This light was situated so that it did not illuminate the stimulus patterns or other objects in the S's visual field. Stimuli were presented only when the $S$ was relatively still, with eyes open, and oriented toward the stimulus. Although the patterns were not visible during the between-stimulus interval, the stimuli were presented at a rate rapid enough to hold the infant's attention for sustained blocks of time. White noise masked any sounds associated with the discharge of the flash tube as well as other extraneous noise.
The $\mathrm{S}$ was subjected to a total of 23 recording sessions between his 21 st and 156th day of life. A session consisted of two blocks of 50 presentations of each of the five patterns. We attempted to replicate the experimental conditions twice during each session, counterbalancing stimulus order in an ABCDEEDCBA manner. In some sessions, however, the $S$ fell asleep or fussed, and only one replication was obtained. Ordinarily, a total of 10 averaged VERs, based on 50 responses each, were recorded in a session. Stimulus order also was varied systematically between sessions.

\section{RESULTS}

A representative sample of averaged VERs to patterned light flashes at different ages is given in Fig. 1. The waveform of VERs obtained in this study is similar to that described in previous studies where diffuse light flashes were used (Ellingson, 1960, 1964; Engel, 1967; Engel \& Benson, 1968; Ferriss et al, 1967; Hrbek et al, 1966). A detailed description of VER waveform, therefore, will not be given here. The effects of pattern and age were most evident in respect to two VER components: the major surface-positive potential (P2) and surface-negative potential (N2), as indicated in Fig. 1, Day 42. The amplitude (in reference to the base line) and latency (in reference to stimulus onset) of the peaks of these potentials in all the data were measured as a function of stimulus pattern and age. Variance analyses were performed on these measures to aid in the interpretation of the data.

The size of checks in the evoking stimulus had a marked influence on the amplitude of $\mathrm{P} 2$, the nature of influence depending on age (Check Size by Age interaction significant at .01 level). The effects of check size were relatively variable up to about the 35th day of age. From this time on, progressively smaller check sizes evoked the greatest responses as $S$ matured (average check subtense 133, $133,86,86,86,43$, and $43 \mathrm{~min}$ of arc for Days $3140,41-50,51-60,61-70,71-80$, $81-90$, and $137-155$, respectively). By approximately the 70th day of age, the relationship between check size and P2 amplitude is described by an inverted U-shaped function. Such a function has been obtained with adult Ss, checks subtending $20-30 \mathrm{~min}$ of arc evoking the greatest amplitude VERs (Harter \& White, 1969; Rietveldt et al, 1967). The variables that might underlie this function have been discussed in these studies and, therefore, will not be considered here. It is interesting to note that the critical check sizes evoking the greatest $\mathrm{P} 2$ response in this study correspond to some degree with the check sizes that infants of comparable 
Fig. 1. Sample of averaged visually evoked responses in an infant (from 21 to 155 days of age) to checkerboard-patterned light flashes [individual checks subtending 0 (blank), 20,43,86, and $133 \mathrm{~min}$ of arc]. The responses obtained on Days 21 and 27 are based on 100 summations (vertical marker indicates 10 microvolts), the remaining responses are based on 50 summations (vertical marker indicates $30 \mathrm{~m}$ icrovolts). Superimposed tracings reflect replications obtained on the same day, except for Days 137-155. The P2 and $\mathrm{N} 2$ components that were measured are indicated on the response to the blank pattern, Day 42. Negativity up. The base line was determined by the average voltage level at trace onset.

ages preferred to fixate (Brennan, Ames, \& Moore, 1966; Karmel, 1969).

The effects of pattern on the amplitude of N2 at different ages also were significant (Check Size by Age interaction significant at .05 level). These effects were similar to those shown with P2 amplitude, although they were more variable, especially before approximately the 70th day of age.

The amplitude of $\mathrm{P} 2$ and $\mathrm{N} 2$ varied significantly $(p<.01)$ and curvilinearly as a function of age: Amplitude progressively increased to a maximum at approximately 65 days and then progressively decreased for the remainder of the experiment. The changes in average VER amplitude, as a function of age, appeared to be inversely related to the variability of the individual responses averaged. Visual inspection of the superimposed individual responses indicated that the variability (1) progressively decreased from approximately the 21 st to the 65 th day of age, possibly due to an increase in the alertness of $S$, and (2) progressively increased from approximately the 65th to the 155th day of age, possibly due to increases in movement artifacts and in amplitude of the background activity in the EEG records.

The latency of both $\mathrm{P} 2$ and $\mathrm{N} 2$ decreased significantly as a function of age $(p<.01)$. The latency of P2 decreased from approximately $160 \mathrm{msec}$ during the $21 \mathrm{st}$ to 30 th days of age to $90 \mathrm{msec}$ during the 137th to 155 th days of age. This function was negatively accelerated, little change in latency occurring after the second month of age. The latency of N2 decreased nearly linearly as a function of age from approximately 250 to $130 \mathrm{msec}$. These changes corroborate earlier studies where similar decreases in latency as a function of age were noted and discussed in detail (Ellingson, 1960, 1964; Engel, 1967: Engel \& Benson, 1968; Ferriss et al, 1967; Hrbek et al, 1966). Check size had

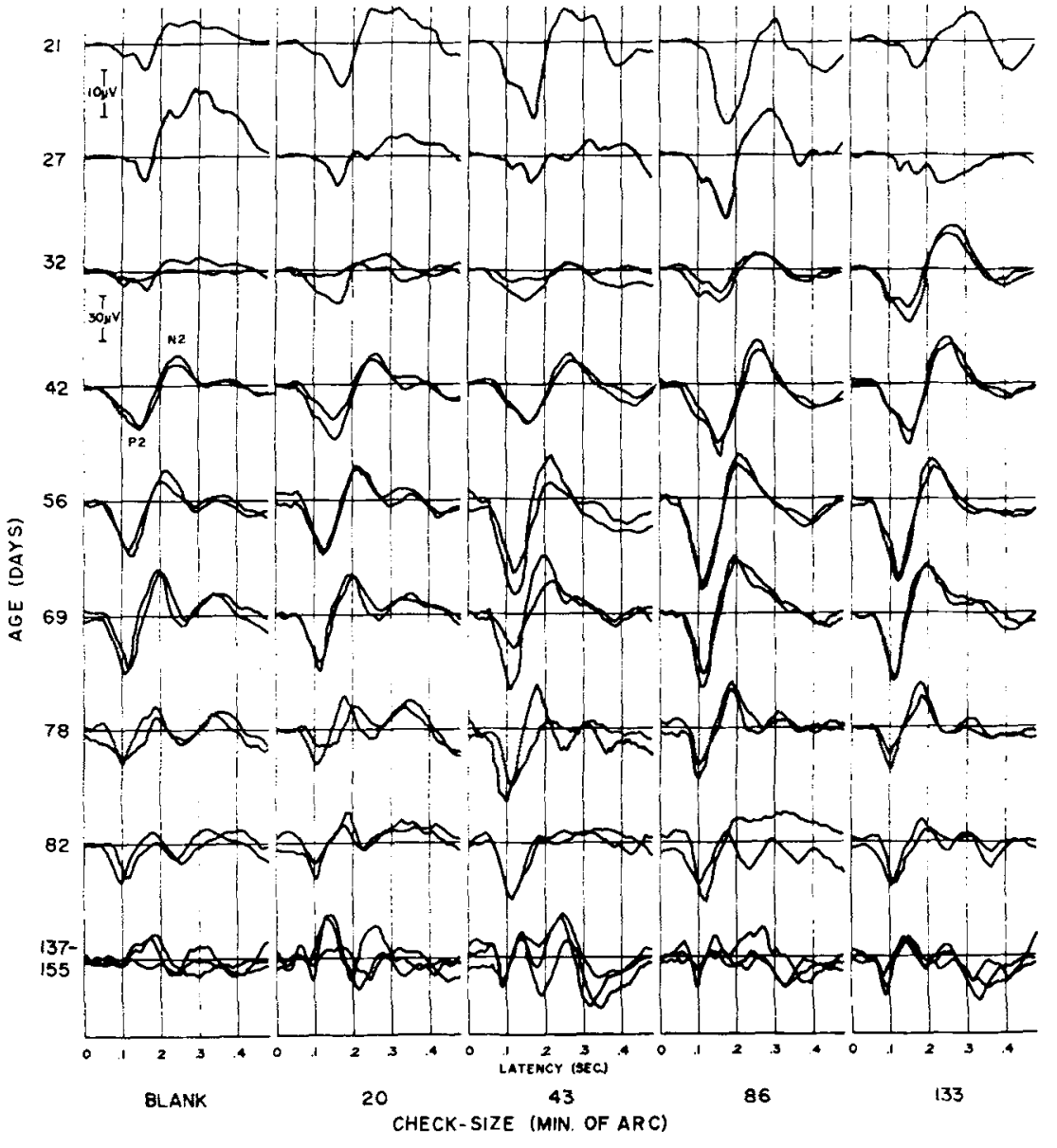

no systematic influence on the latency of either $\mathrm{P} 2$ or $\mathrm{N} 2$.

\section{DISCUSSION}

The unique findings of the present study are (1) that the size of checks in patterned light flashes influence the amplitude of VERs in an infant, and (2) that the effect of check size on VER amplitude varied as a function of age. Our attention, therefore, will be directed toward the possible variables that might underlie these pattern effects. Evidence may be offered that the effects of check size on VER amplitude are a function of visual acuity. An inverted "U"-shaped function between check size and VER amplitude has been noted previously in adults (Rietveld et al, 1967). The nature of this function has been found to vary with eccentricity of retinal stimulation ${ }^{2}$ and refractive error (Harter \& White, 1969), two factors known to influence visual acuity. Decreasing retinal eccentricity and refractive error had the same effect as increasing age in the present study: Progressively smaller check sizes evoked the greatest amplitude VERs. This suggests that the effects of check size at different ages in the present study may reflect changes in visual acuity.
An improvement in visual acuity during infancy has been noted in previous studies employing behavioral measures (Fantz, Ordy, \& Udelf, 1962; Gorman, Gogen, \& Gellis, 1967). These studies indicated visual acuities between $20 / 400$ and $20 / 670$ for the 1st month of life and approximately $20 / 200$ for the 3rd month of life. Data collected at 1 and 3 months of age in the present study were similar to data collected from adults with visua! acuities of about $20 / 500$ (5 diopters induced refractive error) and 20/250 (3 diopters induced refractive error), respectively (Harter \& White, 1969), in terms of the effects of check size on VER amplitude. If these values are taken as an estimate of visual acuity in our $S$, they correspond closely with the behavioral measures.

There are numerous physiological and optical factors that change and develop during infancy that influence the responsiveness of the visual system to patterned stimulation: the visual receptors and central nervous system, refractive error (length of eyeball and curviture of cornea), convergence, conjugation, and accomodation (Hershenson, 1967). Postnatal development of convergence, 
conjugation, and accomodation probably contributed little to the present results since the experimental room was darkened and the visual cues necessary for these mechanisms to function normally were absent. The results most likely are attributable to macular development, which continues through the 4th month of life (Walsh, 1957) and changes in refractive error. Infants are usually hypermetropic (farsighted) at birth since the eyeball is too short for the focal length of the optical mechanisms of the eye; hypermetropia usually decreases with age as the eyeball increases in size (Duke-Elder, 1963). Evidence has been offered that the mean refractive error of newborns is 1.5 diopters (Hershenson, 1967). We have offered evidence that at least 5 diopters of refractive error would be required to account for the effects of check size on VER amplitude in this study at the age of 1 month. This suggests that the apparent improvement in visual acuity with age was primarily due to macular development.

In conclusion, these results indicate that VERs to patterned light flashes give valuable information about the development of mechanisms influencing pattern vision in the human infant. Additional investigations will be required to determine the generality of these data and whether or not they are attributable to the development of mechanisms underlying visual acuity as proposed in this study.

\section{REFERENCES}

$B E R L Y N E, D . E$. The influence of the albedo and complexity of stimuli on visual fixation in the human infant. British Journal of Psychology, 1958, 49, 315-318.

BRIENNAN, W, M., AMIE, E. W., \& MOORE, R. $W$. Age differences in infants' attention to patterns of different complexity. Science, $1966.151,354-356$.

DUKE-LLDER, S. Sy'stems of ophthalmology. Vol. III. Normal and abnormal development Part 1: Embryology. St. Louis: Mosby, 1963. P. 312 .

ELLINGSON, R. J. Cortical electrical responses to visual stimulation in the human infant. Electrocncephalography \& Clinical Neurophysiology, 1960, 12, 663-677.

ELLINGSON, R. J. Studies of the electrical activity of the developing human brain. In $W$. A. Himwich and H. E. Himwich (Eds.), Progress in brain research. Vol. 9. The developing brain. New York: Elsevier, 1964. Pp. 26-53.

IENGEL, R. Electroencephalographic responses to sound and to light in premature and full-term neonates. Lancet, $1967,87,181-186$.

ENGEL, R., \& BENSON, R. C. Estimate of conceptional age by evoked response activity. Biologia Neonatorum, 1968, 12, 201-213.

I:ANTZ, R. L.. ORDY, J. M.. \& UDELI. M. S. Maturation of pattern vision in infants during the first six months. Journal of Comparative \& Physiological Psychology, 1962, 55, 907-917.

IERRISS, G. S., DAVIS, G. D., DORSEN, M. Mcl.. \& HACKETT, F. R. Changes in latency and form of the photically induced average cvoked response in human infants I Icctroencephalography \& Clinical Neurophysiology, 1967, 22, 305-312.

GORMAN, J. J., GOGEN, D. G., \& GELLIS, S. $S$. Testing the visual acuity of infants, In $Y$. Brackbili and G. G. Thompson (Eds.), Behavior in infancy and early childhood. New York: The Free Press, 1967. Pp. 173-178.

HARTER, M. R., \& WHITE, C. T. Effects of contour sharpness and check-size on visually evoked cortical potentials. Vision Research, $1968,8,701-711$.

HARTER, M. R., \& WHITE, C. T. Evoked cortical responses to checkerboard patterns: Effects of check-size as a function of visual acuity. Electroencephalography \& Clinical Neurophysiology, $1969,28,48-54$.

HERSHENSON, M, Development of the perception of form. Psychological Bulletin. $1967,67,326-336$.

HRBEK, A., VITOVA, Z., \& MARES, P. The development of cortical evoked responses to visual stimulation during childhood. Activitas Nervosa, 1966, 8, 39-46.

KARMEL, B. Z. The effects of age, complexity, and amount of contour on pattern preferences in human infants. Journal of Experimental Child Psychology, 1969, 7, 339-354.

RIETVELD, W. J., TORDOIR, W. E.,
HAGINOUW, J. R., LUBBERS, J. A., * SPOOR, Th. A. Visual evoked response to blank and to checkerboard patterned flashes. Acta Physiologica \& Pharmacologica Neerlandia, 1967, 14, 259-285.

SPEHLMANN, $R$. The averaged electrical responses to diffuse and to patterned light in the human. Electroencephalography \& Clinical Neurophysiology, $1965,19,560-569$.

WALSH, F. B. Neuro-ophthalmology. Baltimore: Williams \& Wilkins, 1957. P. 348.

\section{NOTES}

1. This research was supported by funds received from the National Science Foundation under Grants GB-8053 and GB-7324 and from a University of North Carolina institutional grant. Our idea of using VERs to study pattern vision in infants was originally conceptualized with Bernard Z. Karmel and Carroll $T$. White at the Naval Electronics Laboratory Center, San Diego, California. We thank Susan A. Harter, the mother of our subject, for her help in conducting this study.

2. Harter, M. R. Evoked cortical potential correlates of visual acuity and pattern perception in humans. Presented at Southeastern Psychological Association, 1969 (in preparation for publication).

\section{Catecholamine excretion as a function of}

\section{personality ${ }^{1}$}

HARTMUT SCHULZ and HANS STROBACH, Psychological Institute and Pharmacological Institute, University of Düsseldorf, West Germany

An experiment was designed to study the relationship between urine catecholamine excretion and the personality traits of extraversion and neuroticism. Using chance samples of day-urine, we found a significant negative correlation between extraversion and the amount of urine catecholamines. This relationship is modified by smoking habits. The correlation holds only for nonsmokers.

Although it is well established that excretion rates of adrenaline (A) and noradrenaline (NA) are strongly influenced by stressful situational factors and by momentary affective states (Levi, 1967; Schildkraut \& Kety, 1967), there is no good evidence of a correlation with stable, transituational personality traits (Mason, 1968).

Here we show a relationship between catecholamine excretion and extraversion-introversion (E) and neuroticism-stability $(\mathrm{N})$ as measured by the Maudsley Personality Inventory, or MPI (Eysenck, 1959).
On Eysenck's hypothesis, that extraversion is associated with low and unspecific cortical atousal by pathways from the reticular formation (Eysenck, 1967), we assumed that it also would be associated with a low level of peripheral catecholamines, which regulate blood pressure. The linkage is given by experiments that show that the activity of the cells of the reticular formation depends on arterial blood pressure (Baust et al, 1963). The MPI was also used in two other studies of catecholamine output (Levi, 1963; Fine \& Sweeney, 1968), but the results are not clear. Levi (1963), who used peer ratings and MPI scores, presumably on the $\mathbf{N}$ scale, for selecting groups of high and low emotional resistance, did not give any data on the relationship between MPI scores and the excretion of catecholamines. Fine and Sweeney, who tested 27 soldiers under different experimental settings in a 3-day experiment, found a positive relation between MPI-N and the noradrenaline-adrenaline (NA/A) ratio, but the meaning of the ratio (as will be shown later on) is in doubt. They found no significant relation between MPI-E and catecholamine excretion, perhaps because they used 24-h samples instead of day-urine only. 